\title{
Emerging modes of PINK1 signaling: another task for MARK2
}

\author{
Dorthe Matenia $^{1}{ }^{*}$ and Eva M. Mandelkow ${ }^{1,2}$ * \\ ' Max-Planck-Institute for Neurological Research, Hamburg, Germany \\ ${ }^{2}$ German Center for Neurodegenerative Diseases-Center of Advanced European Studies and Research, Bonn, Germany
}

\section{Edited by:}

Jean-Marc Taymans, KU Leuven,

Belgium

\section{Reviewed by:}

Luke Esposito, ProteoTech Inc., USA Björn Spittau,

Albert-Ludwigs-University Freiburg, Germany

\section{*Correspondence:}

Eva M. Mandelkow, German Center for Neurodegenerative

Diseases-Center of Advanced

European Studies and Research,

Ludwig-Erhard-Allee 2,

53175 Bonn, Germany

e-mail: mand@mpasmb.desy.de,

Dorthe Matenia, Max-Planck-Institute for Neurological Research, Hamburg

Outstation, c/o DESY Geb. 25b,

Notkestraße 85, 22607 Hamburg,

Germany

e-mail:matenia@mpasmb.desy.de
PTEN-induced kinase 1 (PINK1) acts at multiple levels to promote mitochondrial health, including regulatory influence on ATP-synthesis, protein quality control, apoptosis, mitochondrial transport, and destiny. PINK1 mutations are linked to Parkinson disease (PD) and mostly result in loss of kinase activity. But the molecular events responsible for neuronal death as well as the physiological targets and regulators of PINK1 are still a matter of debate. This review highlights the recent progress evolving the cellular functions of the cytosolic pool of PINK1 in mitochondrial trafficking and neuronal differentiation. Regulation of PINK1 signaling occurs by mitochondrial processing to truncated forms of PINK1, differentially targeted to several subcellular compartments. The first identified activating kinase of PINK1 is MAP/microtubule affinity regulating kinase 2 (MARK2), which phosphorylates T313, a frequent mutation site linked to PD. Kinases of the MARK2 family perform diverse functions in neuronal polarity, transport, migration, and neurodegeneration such as Alzheimer disease (AD). This new protein kinase signaling axis might provide a link between neurodegenerative processes in AD and PD diseases and opens novel possibilities in targeting pathological signaling processes.

Keywords: PINK1, MARK2, mitochondria, transport, differentiation, neurodegeneration, Alzheimer disease, Parkinson disease

\section{INTRODUCTION}

Many neurodegenerative disorders, such as Alzheimer (AD) and Parkinson disease (PD) show mitochondrial abnormalities during their pathogenesis. Neurons, due to their size and complex geometry, are particularly dependent on the proper functioning and distribution of mitochondria, which are the powerhouses of the cells. Beside ATP production, they perform a variety of functions that are important for cell life and death, including reactive oxygen species (ROS) generation, intracellular calcium homeostasis, and apoptosis. In the nervous system, mitochondrial dynamics are crucial to guarantee long distance delivery and balanced distribution of energy to axons, dendrites and synapses (Jacobson and Duchen, 2004; DiMauro and Schon, 2008). Tau and other microtubule associated proteins promote the assembly and stabilization of neuronal microtubule tracks and ensure microtubule dependent transport. Pathological changes of tau may lead to the breakdown of microtubules observed in $\mathrm{AD}$ while elevated tau on microtubules can compete with motor proteins, resulting in inhibition of traffic (Mandelkow et al., 2004; Dixit et al., 2008). This suggests that a strict regulation is needed to maintain the flow of material. Phosphorylation of tau, especially at the KXGS motifs of the repeat domain, decreases its affinity to microtubules and provides a mechanism for regulating microtubule stability as well as axonal transport (Matenia and Mandelkow, 2009). Enhanced phosphorylation of tau at multiple sites is an early hallmark of $\mathrm{AD}$, followed by abnormal aggregation of tau protein into paired helical filaments (PHFs) and neurofibrillary tangles (NFTs). The microtubule-affinity regulating kinase 2 (MARK2) was originally discovered by its ability to phosphorylate tau protein and related microtubule-associated proteins (MAPs; Drewes et al., 1997; Schwalbe et al., 2013). Furthermore, active MARK2 colocalizes with NFTs in AD brain, and MARK2 target sites on tau are elevated in transgenic mouse models of tauopathy, emphasizing the importance of MARK2 in this disease (Matenia and Mandelkow, 2009). Recently, MARK2 was identified as an upstream regulator of PTEN-induced kinase 1 (PINK1; Matenia et al., 2012). This provides insights into the regulation of mitochondrial trafficking in neurons and a potential link between neurodegenerative processes in $\mathrm{AD}$ and $\mathrm{PD}$.

\section{PTEN-INDUCED KINASE 1}

Familial cases of PD can be caused by mutations in different genes, such as PINK1 or Parkin. PINK1 is a mitochondria-targeted serine/threonine kinase promoting cell survival, particularly under conditions of oxidative/metabolic stress (Valente et al., 2004; Deng et al., 2005; Wood-Kaczmar et al., 2008). In particular, PINK1 regulates mitochondrial transport, morphology, biogenesis, function, calcium buffering capacity, and mitochondrial clearance (Petit et al., 2005; Wood-Kaczmar et al., 2008; Dagda and Chu, 2009; Gandhi et al., 2009; Gegg et al., 2009; Van Laar and Berman, 2009; Matsuda et al., 2010; Narendra et al., 2010; Sun et al., 2012). Most of the reported PD-linked PINK1 mutations result in a loss of kinase activity (Cookson and Bandmann, 2010).

The molecular events responsible for PINK1-induced neuronal death as well as its physiological substrates or regulators are still a matter of debate (Deas etal., 2009; Pogson etal., 
2011). Upon entry to the mitochondria the PINK1 protein is proteolytically cleaved by mitochondrial processing peptidase (MPP) and presenilin-associated rhomboid-like protease (PARL) to produce two N-terminally truncated protein fragments of 54 and $45 \mathrm{kDa}$ without mitochondrial localization sequence (Deas etal., 2009; Narendra etal., 2010; Greene etal., 2012). The cleaved $\triangle \mathrm{N}$-PINK1 forms localize preferentially in the cytosolic instead of the mitochondrial fraction (Lin and Kang, 2008). $\triangle \mathrm{N}$-PINK1 is constitutively degraded in the cytosol by the proteasomal pathway (Yamano and Youle, 2013), indicating that only the mitochondrially targeted PINK $1^{\mathrm{FL}}$ has a cellular function. But expression of $\triangle N$-PINK1 protects neurons against the neurotoxin 1-methyl-4-pheny-1,2,3,6-tertahydropyridin (MPTP). This suggests that the mitochondrial import sequence of PINK1 is not strictly necessary for neuroprotection and that cytosolic targets and signal transduction pathways may be modified by cleaved PINK1 ( $\triangle \mathrm{N}$-PINK1) to affect neuronal survival (Haque et al., 2008). Recent studies validate this hypothesis. PINK1 cleavageproducts localized in the cytosol are degraded by proteasomes but also bind Parkin, repress Parkin translocation to mitochondria and prevent mitophagy (Fedorowicz et al., 2014; Figure 1A). Furthermore, cytosolic $\Delta \mathrm{N}$-PINK1 influences mitochondrial mobility. The kinase enhances anterograde movements of mitochondria, both in dendrites and axons (Matenia et al., 2012; Dagda et al., 2013). However, the mechanisms of these $\Delta \mathrm{N}$-PINK1 functions are mostly unknown. So far only one upstream regulating kinase was identified: MARK2 phosphorylates PINK1 and thereby regulates mitochondrial transport parameters (Matenia et al., 2012). This new signaling axis might help to clarify common mechanisms in neurodegenerative diseases, although future studies are required to understand the exact functional relationship of these kinases.

\section{REGULATION OF PINK1 AND MITOCHONDRIAL MOTILITY IN NEURONS}

Recent studies have investigated the PINK1/Parkin pathway for sensing and selectively eliminating damaged mitochondria from the mitochondrial network. Parkin is a cytoplasmic E3 ubiquitin ligase and can be phosphorylated by PINK1 (Kim et al., 2008). Both proteins cooperate to control mitochondrial clearance, known as mitophagy. Full length PINK1 $\left(\mathrm{PINK}{ }^{\mathrm{FL}}\right.$ ) is stabilized on mitochondria with low membrane potential and recruits cytosolic Parkin, which becomes enzymatically active and initiates the lysosomal degeneration of defective mitochondria via ubiquitination of mitochondrial target proteins (Youle and Narendra, 2011; Grenier et al., 2013; Figure 1B).

Another aspect of PINK1 concerns its role in the regulation of mitochondrial transport in neurons (Wang et al., 2011; Liu et al., 2012; Matenia et al., 2012; Dagda et al., 2013). Mitochondria are transported along microtubules by the motor proteins kinesin (anterograde, toward the microtubule plus ends) and dynein (retrograde). The kinesin-adaptor complex attached to the outer mitochondrial membrane comprises the GTPase Miro, kinesin heavy chain, and the adaptor protein Milton (Goldstein et al., 2008). PINK $1^{\mathrm{FL}}$ is also attached to this complex, and even $\Delta \mathrm{N}$ PINK1 can be targeted to it despite the lack of the mitochondrial targeting sequence (Weihofen et al., 2009). PINK1 ${ }^{\mathrm{FL}}$ phosphorylates the GTPase Miro, and thus induces Parkin-dependent degeneration of Miro. The resulting decrease in mitochondrial movement may represent a quality control mechanism of defective
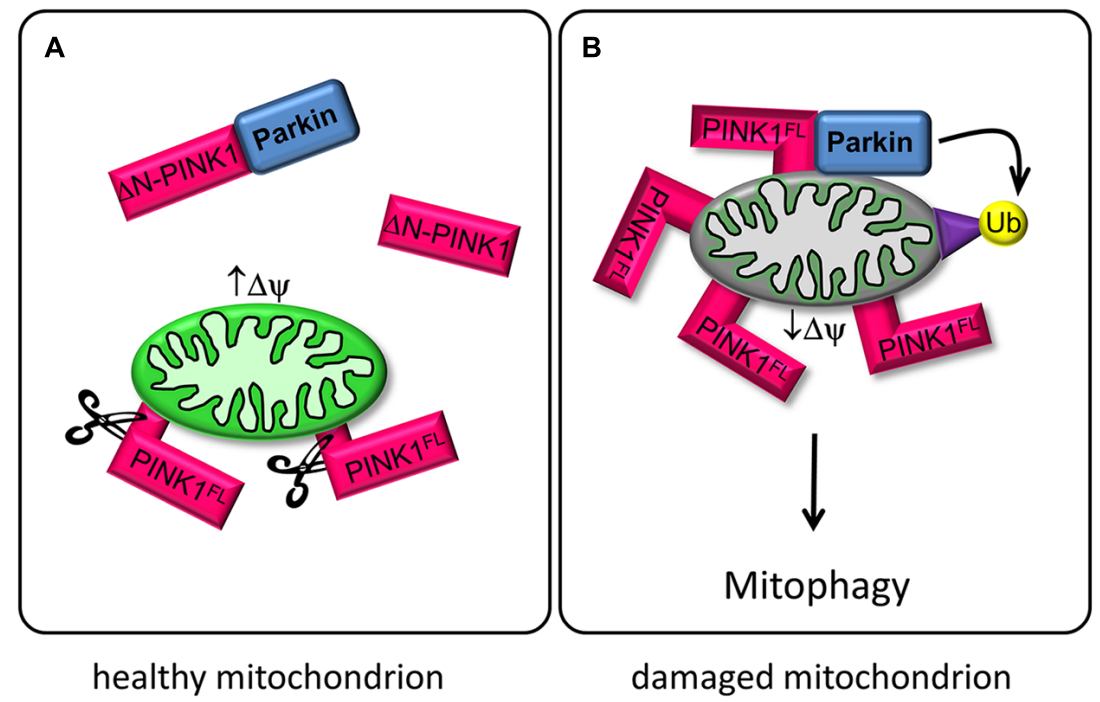

FIGURE 1 | PINK1 and Parkin regulate mitophagy. (A) In healthy mitochondria with high mitochondrial membrane potential $(\uparrow \Delta \Psi)$, $\mathrm{PINK} 1 \mathrm{FL}$ is maintained at low levels by the sequential proteolytic actions of mitochondrial processing peptidase (MPP) and presenilin-associated rhomboid-like protease (PARL; Greene etal., 2012). The resulting $\triangle \mathrm{N}$-PINK1 is partially located in the cytosol and interacts directly with Parkin, thereby preventing Parkin-mediated mitophagy
(Fedorowicz etal., 2014). (B) Upon mitochondrial depolarization $(\downarrow \Delta \Psi)$ PINK1 $1 F$ is stabilized and selectively accumulates in the outer membrane of defective mitochondria with its kinase domain facing the cytoplasm. This accumulation is a crucial signal for Parkin recruitment to impaired mitochondria, promoting ubiquitination of mitochondrial outer membrane proteins and subsequent disposal of the damaged organelle (Narendra etal., 2010). 
mitochondria (Wang etal., 2011). Active mitochondria with a high membrane potential tend to cause cleavage of PINK1 ${ }^{\mathrm{FL}}$ to $\triangle \mathrm{N}$-PINK1, which is released to the cytoplasm, where it is not only destined for degradation by the proteasome but also binds to Parkin (Miller and Sheetz, 2004; Figure 1A). The interaction of cytosolic $\triangle \mathrm{N}$-PINK1 with Parkin represses Parkin translocation to the mitochondria and subsequent mitophagy (Fedorowicz et al., 2014). The question is therefore how the different isoforms of PINK1 become active.

Only few studies have examined the regulation of PINK1 and its consequences for mitochondria. The serine/threonine kinase MARK2 phosphorylates PINK1, activates the kinase activity of $\triangle \mathrm{N}-\mathrm{PINK} 1$ (with regard to the artificial substrate histone $\mathrm{H} 4$ ) and enhances protein stability of both, PINK ${ }^{\mathrm{FL}}$ and $\Delta \mathrm{N}-\mathrm{PINK} 1$, arguing for a physiological relevance of this kinase-substrate interaction. The primary phosphorylation site is threonine 313 (T313; Matenia etal., 2012). This residue is mutated to a non-phosphorylatable form (T313M) in a frequent variant of PD (Mills etal., 2008). Residue T313 is located in $\beta$-strand 5 of PINK1 (based on a structural model of PINK1 by SwissModel using CaMK1 as a structure template; Matenia etal., 2012). Phosphorylation of this residue could therefore result in the interaction of this strand with helix $\mathrm{C}$ which is part of the scaffold that fixes the Mg-ATP underneath the P-loop. Stabilization of this part of the catalytic domain is a requirement for the activity of the kinase. In fact, mutation of T313 to glutamate further enhances the phosphorylation and activation of $\triangle \mathrm{N}$-PINK1 by MARK2, suggesting that this residue could be a priming phosphorylation site, changing the conformation of the kinase and preparing it for further modifications.

The importance of the PINK1 phosphorylation site T313 is further emphasized by the fact that expression of PINK $1^{\mathrm{T} 313 \mathrm{M}}$ causes severe toxicity for cells. $\Delta \mathrm{N}$-PINK $1^{\mathrm{T} 313 \mathrm{M}}$ leads to abnormal mitochondrial accumulation in the cell soma, whereas PINK1 ${ }^{\text {FL/T313M }}$ causes degradation of mitochondria. Within neurons endogenous PINK1 and MARK2 colocalize partly on mitochondria, especially in axons and dendrites, changing mitochondrial transport parameters (mitochondrial density and movement direction in axons). MARK2 interacts with and preferentially phosphorylates the cytosolic $\triangle \mathrm{N}$-PINK1, thereby increasing its kinase activity and promoting anterograde mitochondrial motility (Matenia et al., 2012). Consistent with this, a high membrane potential enhances the anterograde transport of mitochondria (Miller and Sheetz, 2004) and also promotes the proteolysis of PINK1 ${ }^{\mathrm{FL}}$ into $\triangle$ N-PINK1 (Narendra et al., 2010), thereby inhibiting Parkindependent mitophagy of active mitochondria (Figure 2). Phosphorylation and activation of $\triangle \mathrm{N}$-PINK1 by MARK2 possibly enhances the stability of the mitochondrial transport-complex and ensures the supply of energy at the growth cone. Conversely, retrograde transport is favored for mitochondria with low membrane potential destined for mitophagy (Jin et al., 2010). In this case the cleavage of PINK1 ${ }^{\mathrm{FL}}$ is inhibited. Thus, Parkin decorated mitochondria assemble as large clusters primarily in the lysosome-rich perinuclear area (Narendra et al., 2010). This effect is influenced by MARK2 (Matenia et al., 2012). MARK2 could phosphorylate PINK $1^{\mathrm{FL}}$, consequently enhance the binding and possibly the phosphorylation of Parkin and Miro by PINK1 ${ }^{\mathrm{FL}}$. This results in accumulation of mitochondria around the perinuclear region and suggests that failure of the MARK2-PINK1 signaling cascade could contribute to PD. Thus, our study revealed the existence of two cellular pools of PINK1 that differently modify and regulate mitochondrial movement direction.

\section{PINK1, MARK2, AND DIFFERENTIATION}

$\mathrm{MAP} /$ microtubule affinity regulating kinase 2 is involved in several regulatory processes of the cell such as determination of polarity, cell cycle control, intracellular signal transduction, transport, and cytoskeletal stability (Matenia and Mandelkow, 2009). MARK2 and its homolog par-1 (for "partition defective") belongs to a set of conserved proteins in Drosophila and Caenorhabditis elegans, which are essential for cellular polarity, with roles in establishing the embryonic body axis and in maintaining cell differentiation (Kemphues et al., 1988; Tomancak et al., 2000). The par-1-dependent cell polarization is based on a tight network of cross-reactive and feedback interactions of the par proteins, other regulators of polarity and the cytoskeleton (Munro, 2006). MARK/par-1 is a central player in localization of the cell polarity proteins. In mammalian epithelial cells the overexpression of inactive MARK2 disturbs the polarity, suggesting a similar mechanism of governing polarization (Böhm et al., 1997).

Microtubules are important determinants of cell polarity. MARK2 plays a significant role in axon formation, which requires dynamic instability of microtubules (Biernat et al., 2002). This is in part related to the phosphorylation of axonal Tau protein in its "repeat domain" which decreases its affinity for microtubules. The reduction of MARK2 via RNA interference (RNAi) induces multiple axons in hippocampal neurons, whereas enhanced MARK2 expression inhibits axon formation altogether (Chen et al., 2006). Following the establishment of an axon MARK2 promotes its elongation (Uboha et al., 2007). In dendrites, the predominant MAP is MAP2 which has a similar repeat domain as Tau and can also be phosphorylated by MARK2 (Illenberger et al., 1996). In this case MARK2 inhibits the development of dendrites in hippocampal neurons through phosphorylation of MAP2. In particular, MARK2 shortens the length and decreases branching of dendrites (Terabayashi et al., 2007).

Interestingly, transient expression of $\Delta \mathrm{N}$-PINK1 promotes dendritic outgrowth and neurite length in dopaminergic midbrain neurons. This effect seems to be kinase dependent, since a kinase deficient mutant of PINK1 fails to influence neurite length. The action of $\Delta N$-PINK1 on neurite length was not related to its activity at mitochondria, since an outer mitochondria membrane (OMM)-targeted $\triangle \mathrm{N}$-PINK1 construct, which exhibits cytosolic localization, failed to enhance neuronal differentiation. These data indicate divergent roles for cytosolic and mitochondrial targeted forms of PINK1. Furthermore, PINK1 deficiency reduces dendritic length of primary neurons isolated from PINK1 knockout mice. To clarify the mechanism underlying the regulation of neurite outgrowth induced by cytosolic $\Delta \mathrm{N}-\mathrm{PINK} 1$, Dagda et al. (2013) examined the expression of various neuronal differentiation proteins as a function of PINK1. PINK1 increases levels of MAP2 and activates protein kinase A (PKA)-regulated signaling 


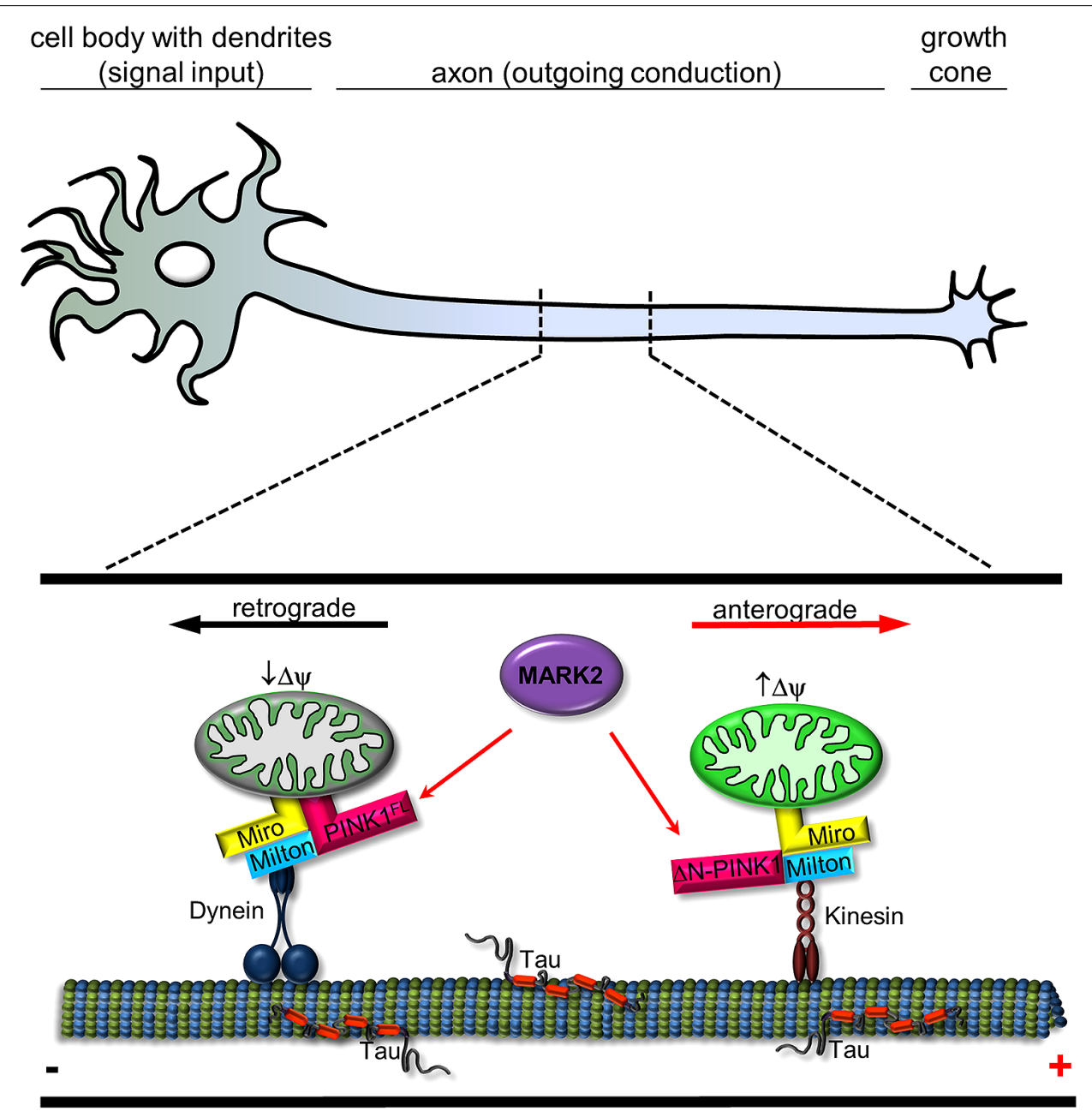

FIGURE 2 | Schematic representation of interplay between MARK2 and PINK1FL/ $\mathbf{N}$-PINK1 to regulate mitochondrial transport. In a healthy neuron, mitochondria are carried along by motor proteins dynein (retrograde) and kinesin (anterograde). PINK1 is a molecular switch that changes the probability between anterograde and retrograde mitochondrial transport. Transport direction of neuronal mitochondria is regulated by PINK1 cleavage and binding/phosphorylation by MARK2. Kinesin motors are linked to mitochondria by adaptor proteins like Miro and Milton (Weihofen et al., 2009) and regulate in association with $\triangle \mathrm{N}$-PINK1 the anterograde movement (red arrow; Matenia et al., 2012; Dagda et al., 2013), whereas Miro also has an effect on dynein-mediated retrograde movement (Russo et al., 2009; black arrow). Active mitochondria tend to cause cleavage of PINK1 FL (Narendra et al., 2010), which is phosphorylated at Thr-313 by MARK2 (Matenia etal., 2012); both events promote anterograde movement by kinesin. Retrograde movement by dynein is promoted by PINK $1{ }^{\mathrm{FL}}$ and further increased by MARK2 (Matenia etal., 2012). pathways. Since MAP2 is an anchoring protein of PKA in dendrites (Obar et al., 1989; Harada et al., 2002), this data suggests that PINK1 is an upstream regulatory kinase of this pathway to influence dendritic morphology. On the other hand, the ability of microtubule-associated PKA to promote elongation of dendrites is independent of MAP2 phosphorylation. This suggests other proteins in close proximity to the microtubule cytoskeleton are involved in this process (Huang et al., 2013). Since KXGS is not only a kinase consensus motif for targets of MARK2 but also of PKA, both kinases share some substrate preferences (Drewes et al., 1997). The microtubule binding affinity of tau as well as that of doublecortin (Dcx) is regulated via phosphorylation by MARK2 and PKA (Drewes et al., 1997; Schaar et al., 2004). This provides the clue, that MARK2 signaling pathways could be involved in $\triangle \mathrm{N}$-PINK1 mediated neurite outgrowth regulation.
Due to substrate competition $\triangle \mathrm{N}$-PINK1 could inhibit MARK2 by binding, thereby enhancing dendritic length.

\section{CONCLUSION AND OUTLOOK}

This review summarizes and evaluates recent findings in PINK1 biology and focuses on emerging aspects concerning the novel role of cytosolic $\triangle \mathrm{N}$-PINK1 that has not yet received adequate attention as compared to mitochondrial PINK $1^{\mathrm{FL}}$. In the case of mitochondria the full-length PINK1 regulates the transport and clearance of defective mitochondria through phosphorylation of Miro and recruitment of Parkin, respectively (Figure 1). These protective activities of PINK $1^{\mathrm{FL}}$ are dependent on its localization at the mitochondrial surface and have been studied extensively. But even the $\mathrm{N}$-terminally truncated enzyme $\Delta \mathrm{N}$-PINK1 lacking the mitochondrial localization signal can be found in close proximity 
to mitochondria, probably via binding to mitochondrial membrane localized protein adaptor complexes (Figure 2), controlling their health status and distribution. Beside this task, $\Delta \mathrm{N}$-PINK1 released from mitochondria via proteolytic cleavage by mitochondrial enzymes shows neurite promoting activity. This outgrowth effect was specific to dendrites as axonal length did not change significantly (Tieu and Xia, 2013).

Microtubule-affinity regulating kinase 2 , the upstream regulator of $\triangle \mathrm{N}$-PINK1 and PINK-1 ${ }^{\mathrm{FL}}$, activates and regulates a diverse range of cellular activities and participates in several signaling cascades. Since the discovery of MARK2 as a kinase of Tau and MAP2 (Drewes et al., 1997), several other substrates have been identified. Some of these affect mitochondrial transport, presumably by regulating the affinity to microtubules (Thies and Mandelkow, 2007; Pluciñska etal., 2012). Neurons are particularly dependent on mitochondrial function, so disrupting the transport of these organelles can cause neurological disease (Schon and Przedborski, 2011). The vulnerability results from the high metabolic demands of neurons, their dependence on proper calcium handling and their susceptibility to local ROS signaling, processes in which mitochondria are critically involved. In the context of transport PINK1 acts as a molecular switch between anterograde and retrograde mitochondrial transport. As mentioned above, transport direction is regulated by PINK1 cleavage depending on the mitochondrial membrane potential (Figure 1) and PINK1 binding/phosphorylation by MARK2 (Figure 2; Matenia et al., 2012). Since MARK2 is an upstream regulatory component in PINK1 signaling, this extends the complexity of its biological function. Mitochondria are enriched at synapses and play a critical role in both pre- and post-synaptic functions (Hollenbeck, 2005). Accurate regulation of mitochondrial motility and maintenance of neuronal plasticity are closely related.

Increasing evidence implicates that dysfunction of kinase activities and phosphorylation pathways are involved in the pathogenesis of neurodegenerative diseases. PINK1 mutations linked to $\mathrm{PD}$ are mostly accompanied by loss of kinase activity; therefore an effective therapy would have to replace functional PINK1signaling. The limiting factor is that the details of the PINK1 signaling network are not yet fully elucidated. An initial step in the right direction is the identification and characterization of a PINK1/Parkin independent mitophagy pathway (Allen et al., 2013). Selective induction of mitophagy could prove beneficial as a potential therapy for several neurodegenerative diseases in which mitochondrial clearance is advantageous.

\section{ACKNOWLEDGMENTS}

We thank Eckhard Mandelkow for suggestions and stimulating discussions. The project was supported in part by the Deutsche Forschungsgemeinschaft, Bundesministerium für Bildung und Forschung (Kompetenznetz Degenerativer Demenzen), and European Union (FP7-MEMOSAD project).

\section{REFERENCES}

Allen, G. F., Toth, R., James, J., and Ganley, I. G. (2013). Loss of iron triggers PINK1/Parkin-independent mitophagy. EMBO Rep. 14, 1127-1135. doi: 10.1038/embor.2013.168

Biernat, J., Wu, Y. Z., Timm, T., Zheng-Fischhöfer, Q., Mandelkow, E., Meijer, L., et al. (2002). Protein kinase MARK/PAR-1 is required for neurite outgrowth and establishment of neuronal polarity. Mol. Biol. Cell 13, 4013-4028. doi: 10.1091/mbc.02-03-0046

Böhm, H., Brinkmann, V., Drab, M., Henske, A., and Kurzchalia, T. V. (1997). Mammalian homologues of C. elegans PAR-1 are asymmetrically localized in epithelial cells and may influence their polarity. Curr. Biol. 7, 603-606. doi: 10.1016/S0960-9822(06)00260-0

Chen, Y. M., Wang, Q. J., Hu, H. S., Yu, P. C., Zhu, J., Drewes, G., et al. (2006). Microtubule affinity-regulating kinase 2 functions downstream of the PAR3/PAR-6/atypical PKC complex in regulating hippocampal neuronal polarity. Proc. Natl. Acad. Sci. U.S.A. 103, 8534-8539. doi: 10.1073/pnas.0509955103

Cookson, M. R., and Bandmann, O. (2010). Parkinson's disease: insights from pathways. Hum. Mol. Genet. 19, R21-R27. doi: 10.1093/hmg/ddq167

Dagda, R. K., and Chu, C. T. (2009). Mitochondrial quality control: insights on how Parkinson's disease related genes PINK1, parkin, and Omi/HtrA2 interact to maintain mitochondrial homeostasis. J. Bioenerg. Biomembr. 41, 473-479. doi: 10.1007/s10863-009-9255-1

Dagda, R. K., Pien, I., Wang, R., Zhu, J., Wang, K. Z., Callio, J., et al. (2013). Beyond the mitochondrion: cytosolic PINK1 remodels dendrites through Protein Kinase A. J. Neurochem. doi: 10.1111/jnc.12494 [Epub ahead of print].

Deas, E., Plun-Favreau, H., and Wood, N. W. (2009). PINK1 function in health and disease. EMBO Mol. Med. 1, 152-165. doi: 10.1002/emmm.200900024

Deng, H., Jankovic, J., Guo, Y., Xie, W., and Le, W. (2005). Small interfering RNA targeting the PINK1 induces apoptosis in dopaminergic cells SH-SY5Y. Biochem. Biophys. Res. Commun. 337, 1133-1138. doi: 10.1016/j.bbrc.2005.09.178

DiMauro, S., and Schon, E. A. (2008). Mitochondrial disorders in the nervous system. Annu. Rev. Neurosci. 31, 91-123. doi: 10.1146/annurev.neuro.30.051606 094302

Dixit, R., Ross, J. L., Goldman, Y. E., and Holzbaur, E. L. (2008). Differential regulation of dynein and kinesin motor proteins by tau. Science 319, 1086-1089. doi: 10.1126/science.1152993

Drewes, G., Ebneth, A., Preuss, U., Mandelkow, E. M., and Mandelkow, E. (1997). MARK, a novel family of protein kinases that phosphorylate microtubuleassociated proteins and trigger microtubule disruption. Cell 89, 297-308. doi: 10.1016/S0092-8674(00)80208-1

Fedorowicz, M. A., de Vries-Schneider, R. L., Rüb, C., Becker, D., Huang, Y., Zhou, C., et al. (2014). Cytosolic cleaved PINK1 represses Parkin translocation to mitochondria and mitophagy. EMBO Rep. 15, 86-93. doi: 10.1002/embr.201337294

Gandhi, S., Wood-Kaczmar, A., Yao, Z., Plun-Favreau, H., Deas, E., Klupsch, K., etal. (2009). PINK1-associated Parkinson's disease is caused by neuronal vulnerability to calcium-induced cell death. Mol. Cell 33, 627-638. doi: 10.1016/j.molcel.2009.02.013

Gegg, M. E., Cooper, J. M., Schapira, A. H., and Taanman, J. W. (2009). Silencing of PINK1 expression affects mitochondrial DNA and oxidative phosphorylation in dopaminergic cells. PLoS ONE 4:e4756. doi: 10.1371/journal.pone.0004756

Goldstein, A. Y., Wang, X., and Schwarz, T. L. (2008). Axonal transport and the delivery of pre-synaptic components. Curr. Opin. Neurobio. 18, 495-503. doi: 10.1016/j.conb.2008.10.003

Greene, A. W., Grenier, K., Aguileta, M. A., Muise, S., Farazifard, R., Haque, M. E., etal. (2012). Mitochondrial processing peptidase regulates PINK1 processing, import and Parkin recruitment. EMBO Rep. 13, 378-385. doi: 10.1038/embor.2012.14

Grenier, K., McLelland, G. L., and Fon, E. A. (2013). Parkin- and PINK1-dependent mitophagy in neurons: will the real pathway please stand up? Front. Neurol. 4:100 doi: 10.3389/fneur.2013.00100

Haque, M. E., Thomas, K. J., D’Souza, C., Callaghan, S., Kitada, T., Slack, R. S., et al. (2008). Cytoplasmic Pink1 activity protects neurons from dopaminergic neurotoxin MPTP. Proc. Natl. Acad. Sci. U.S.A. 105, 1716-1721. doi: 10.1073/pnas.0705363105

Harada, A., Teng, J., Takei, Y., Oguchi, K., and Hirokawa, N. (2002). MAP2 is required for dendrite elongation, PKA anchoring in dendrites, and proper PKA signal transduction. J. Cell Biol. 158, 541-549. doi: 10.1083/jcb.200110134

Hollenbeck, P. J. (2005). Mitochondria and neurotransmission: evacuating the synapse. Neuron 47, 331-333. doi: 10.1016/j.neuron.2005.07.017

Huang, Y. A., Kao, J. W., Tseng, D. T., Chen, W. S., Chiang, M. H., and Hwang, E. (2013). Microtubule-associated type II protein kinase A is important for neurite elongation. PLoS ONE 8:e73890. doi: 10.1371/journal.pone.0073890

Illenberger, S., Drewes, G., Trinczek, B., Biernat, J., Meyer, H. E., Olmsted, J. B., et al. (1996). Phosphorylation of microtubule-associated proteins MAP2 
and MAP4 by the protein kinase p110mark. Phosphorylation sites and regulation of microtubule dynamics. J. Biol. Chem. 271, 10834-10843. doi: 10.1074/jbc.271.18.10834

Jacobson, J., and Duchen, M. R. (2004). Interplay between mitochondria and cellular calcium signaling. Mol. Cell. Biochem. 256-257, 209-228. doi: 10.1023/B:MCBI.0000009869.29827.df

Jin, S. M., Lazarou, M., Wang, C., Kane, L. A., Narendra, D. P., and Youle, R. J. (2010). Mitochondrial membrane potential regulates PINK1 import and proteolytic destabilization by PARL. J. Cell Biol. 191, 933-942. doi: 10.1083/jcb.2010 08084

Kemphues, K. J., Priess, J. R., Morton, D. G., and Cheng, N. S. (1988). Identification of genes required for cytoplasmic localization in early C. elegans embryos. Cell 52, 311-320. doi: 10.1016/S0092-8674(88)80024-2

Kim, Y., Park, J., Kim, S., Song, S., Kwon, S. K., Lee, S. H., et al. (2008). PINK1 controls mitochondrial localization of Parkin through direct phosphorylation. Biochem. Biophys. Res. Commun. 377, 975-980. doi: 10.1016/j.bbrc.2008. 10.104

Lin, W., and Kang, U. J. (2008). Characterization of PINK1 processing, stability, and subcellular localization. J. Neurochem. 106, 464-474. doi: 10.1111/j.14714159.2008.05398.x

Liu, S., Sawada, T., Lee, S., Yu, W., Silverio, G., Alapatt, P., et al. (2012). Parkinson's disease-associated kinase PINK1 regulates Miro protein level and axonal transport of mitochondria. PLoS Genet. 8:e1002537. doi: 10.1371/journal.pgen.1002537

Mandelkow, E.-M., Thies, E., Trinczek, B., Biernat, J., and Mandelkow, E. (2004). MARK/PAR1 kinase is a regulator of microtubule-dependent transport in axons. J. Cell Biol. 167, 99-110. doi: 10.1083/jcb.200401085

Matenia, D., Hempp, C., Timm, T., Eikhof, A., and Mandelkow, E. M. (2012). Microtubule affinity-regulating kinase 2 (MARK2) turns on phosphatase and tensin homolog (PTEN)-induced kinase 1 (PINK1) at Thr-313, a mutation site in Parkinson disease: effects on mitochondrial transport. J. Biol. Chem. 287, 8174-8186. doi: 10.1074/jbc.M111.262287

Matenia, D., and Mandelkow, E. M. (2009). The tau of MARK: a polarized view of the cytoskeleton. Trends Biochem. Sci. 34, 332-342. doi: 10.1016/j.tibs.2009.03.008

Matsuda, N., Sato, S., Shiba, K., Okatsu, K., Saisho, K., Gautier, C. A., et al. (2010) PINK1 stabilized by mitochondrial depolarization recruits Parkin to damaged mitochondria and activates latent Parkin for mitophagy. J. Cell Biol. 189, 211-221. doi: $10.1083 /$ jcb. 200910140

Miller, K. E., and Sheetz, M. P. (2004). Axonal mitochondrial transport and potential are correlated. J. Cell Sci. 117(Pt 13), 2791-2804. doi: 10.1242/jcs.01130

Mills, R. D., Sim, C. H., Mok, S. S., Mulhern, T. D., Culvenor, J. G., and Cheng, H. C. (2008). Biochemical aspects of the neuroprotective mechanism of PTEN-induced kinase-1 (PINK1). J. Neurochem. 105, 18-33. doi: 10.1111/j.1471-4159.2008.05249.x

Munro, E. M. (2006). PAR proteins and the cytoskeleton: a marriage of equals. Curr. Opin. Cell Biol. 18, 86-94. doi: 10.1016/j.ceb.2005.12.007

Narendra, D. P., Jin, S. M., Tanaka, A., Suen, D. F., Gautier, C. A., Shen, J., et al. (2010) PINK1 is selectively stabilized on impaired mitochondria to activate Parkin. PLoS Biol. 8:e1000298. doi: 10.1371/journal.pbio.1000298

Obar, R. A., Dingus, J., Bayley, H., and Vallee, R. B. (1989). The RII subunit of cAMP-dependent protein kinase binds to a common amino-terminal domain in microtubule-associated proteins 2A, 2B, and 2C. Neuron 3, 639-645. doi: 10.1016/0896-6273(89)90274-2

Petit, A., Kawarai, T., Paitel, E., Sanjo, N., Maj, M., Scheid, M., et al. (2005). Wildtype PINK1 prevents basal and induced neuronal apoptosis, a protective effect abrogated by Parkinson disease-related mutations. J. Biol. Chem. 280, 3402534032. doi: 10.1074/jbc.M505143200

Pluciñska, G., Paquet, D., Hruscha, A., Godinho, L., Haass, C., Schmid, B., et al (2012). In vivo imaging of disease-related mitochondrial dynamics in a vertebrate model system. J. Neurosci. 32, 16203-16212. doi: 10.1523/JNEUROSCI.132712.2012

Pogson, J. H., Ivatt, R. M., and Whitworth, A. J. (2011). Molecular mechanisms of PINK1-related neurodegeneration. Curr. Neurol. Neurosci. Rep. 11, 283-290. doi: 10.1007/s11910-011-0187-x

Russo, G. J., Louie, K., Wellington, A., Macleod, G. T., Hu, F., Panchumarthi, S., et al. (2009). Drosophila Miro is required for both anterograde and retrograde axonal mitochondrial transport. J. Neurosci. 29, 5443-5455. doi: 10.1523/JNEUROSCI.5417-08.2009
Schaar, B. T., Kinoshita, K., and McConnell, S. K. (2004). Doublecortin microtubule affinity is regulated by a balance of kinase and phosphatase activity at the leading edge of migrating neurons. Neuron 41, 203-213. doi: 10.1016/S08966273(03)00843-2

Schon, E. A., and Przedborski, S. (2011). Mitochondria: the next (neurode) generation. Neuron 70, 1033-1053. doi: 10.1016/j.neuron.2011.06.003

Schwalbe, M., Biernat, J., Bibow, S., Ozenne, V., Jensen, M. R., Kadavath, H., et al. (2013). Phosphorylation of human tau protein by microtubule affinity-regulating kinase 2. Biochemistry 52, 9068-9079. doi: 10.1021/bi401266n

Sun, Y., Vashisht, A. A., Tchieu, J., Wohlschlegel, J. A., and Dreier, L. (2012). Voltagedependent anion channels (VDACs) recruit Parkin to defective mitochondria to promote mitochondrial autophagy. J. Biol. Chem. 287, 40652-40660. doi: 10.1074/jbc.M112.419721

Terabayashi, T., Itoh, T. J., Yamaguchi, H., Yoshimura, Y., Funato, Y., Ohno, S., et al. (2007). Polarity-regulating kinase partitioning-defective $1 /$ microtubule affinityregulating kinase 2 negatively regulates development of dendrites on hippocampal neurons. J. Neurosci. 27, 13098-13107. doi: 10.1523/JNEUROSCI.3986-07. 2007

Thies, E., and Mandelkow, E. M. (2007). Missorting of tau in neurons causes degeneration of synapses that can be rescued by the kinase MARK2/Par-1. J. Neurosci. 27, 2896-2907. doi: 10.1523/JNEUROSCI.4674-06.2007

Tieu, K., and Xia, X. G. (2013). Cytosolic PINK1 escapes from mitochondria to promote dendritic outgrowth. J. Neurochem. 128, 787-789. doi: 10.1111/jnc. 12529

Tomancak, P., Piano, F., Riechmann, V., Gunsalus, K. C., Kemphues, K. J., and Ephrussi, A. (2000). A Drosophila melanogaster homologue of Caenorhabditis elegans par-1 acts at an early step in embryonic-axis formation. Nat. Cell Biol. 2, 458-460. doi: 10.1038/35017101

Uboha, N. V., Flajolet, M., Nairn, A. C., and Picciotto, M. R. (2007). A calciumand calmodulin-dependent kinase Ialpha/microtubule affinity regulating kinase 2 signaling cascade mediates calcium-dependent neurite outgrowth. J. Neurosci. 27, 4413-4423. doi: 10.1523/JNEUROSCI.0725-07.2007

Valente, E. M., Salvi, S., Ialongo, T., Marongiu, R., Elia, A. E., Caputo, V., et al. (2004). PINK1 mutations are associated with sporadic early-onset parkinsonism. Ann. Neurol. 56, 336-341. doi: 10.1002/ana.20256

Van Laar, V. S., and Berman, S. B. (2009). Mitochondrial dynamics in Parkinson's disease. Exp. Neurol. 218, 247-256. doi: 10.1016/j.expneurol.2009.03.019

Wang, X., Winter, D., Ashrafi, G., Schlehe, J., Wong, Y. L., Selkoe, D., et al. (2011). PINK1 and Parkin target Miro for phosphorylation and degradation to arrest mitochondrial motility. Cell 147, 893-906. doi: 10.1016/j.cell.2011.10.018

Weihofen, A., Thomas, K. J., Ostaszewski, B. L., Cookson, M. R., and Selkoe, D. J. (2009). Pink1 forms a multiprotein complex with Miro and Milton, linking Pink1 function to mitochondrial trafficking. Biochemistry 48, 2045-2052. doi: $10.1021 /$ bi8019178

Wood-Kaczmar, A., Gandhi, S., Yao, Z., Abramov, A. Y., Miljan, E. A., Keen, G., et al. (2008). PINK1 is necessary for long term survival and mitochondrial function in human dopaminergic neurons. PLOS ONE 3:e2455. doi: 10.1371/journal.pone.0002455

Yamano, K., and Youle, R. J. (2013). PINK1 is degraded through the N-end rule pathway. Autophagy 9, 1758-1769. doi: 10.4161/auto.24633

Youle, R. J., and Narendra, D. P. (2011). Mechanisms of mitophagy. Nat. Rev. Mol. Cell Biol. 12, 9-14. doi: 10.1038/nrm3028

Conflict of Interest Statement: The authors declare that the research was conducted in the absence of any commercial or financial relationships that could be construed as a potential conflict of interest.

Received: 26 February 2014; accepted: 19 April 2014; published online: 08 May 2014. Citation: Matenia D and Mandelkow EM (2014) Emerging modes of PINK1 signaling: another task for MARK2. Front. Mol. Neurosci. 7:37. doi: 10.3389/fnmol.2014.00037 This article was submitted to the journal Frontiers in Molecular Neuroscience. Copyright (C) 2014 Matenia and Mandelkow. This is an open-access article distributed under the terms of the Creative Commons Attribution License (CC BY). The use, distribution or reproduction in other forums is permitted, provided the original author(s) or licensor are credited and that the original publication in this journal is cited, in accordance with accepted academic practice. No use, distribution or reproduction is permitted which does not comply with these terms. 